\title{
A COMPUTATIONAL METHOD FOR FREE TIME OPTIMAL CONTROL PROBLEMS, WITH APPLICATION TO MAXIMIZING THE RANGE OF AN AIRCRAFT-LIKE PROJECTILE
}

\author{
K. L. TEO ${ }^{1}$, G. JEPPS ${ }^{2}$, E.J. MOORE ${ }^{3}$ AND S. HAYES ${ }^{3}$
}

(Received 25 March 1985; revised 7 December 1985)

\begin{abstract}
A class of non-standard optimal control problems is considered. The non-standard feature of these optimal control problems is that they are of neither fixed final time nor of fixed final state. A method of solution is devised which employs a computational algorithm based on control parametrization techniques. The method is applied to the problem of maximizing the range of an aircraft-like gliding projectile with angle of attack control.
\end{abstract}

\section{Introduction}

A class of optimal control problems which frequently occurs in practice has the non-standard feature that they are of neither fixed final time nor of fixed final state. These problems arise in attempting to maximize the range of an aircraft-like gliding projectile when angle of attack is considered as the control variable. This paper describes a computational algorithm, devised to solve such problems, and gives an example of its application to determining the maximum range of a gliding projectile.

The technique employed is developed from that used in [2], [5], [6], [9]-[13], [15], and [18]. This technique is known as the control parametrization technique. It involves the approximation of the control variables by piecewise constant

\footnotetext{
${ }^{1}$ Department of Industrial and Systems Engineering, National University of Singapore, Kent Ridge, Singapore 0511, Singapore.

${ }^{2}$ Weapons Systems Research Laboratory, Defence Science and Technology Organisation, Department of Defence, GPO Box 2151, Adelaide, South Australia 5001, Australia.

${ }^{3}$ Department of Applied Mathematics, University of New South Wales, P. O. Box 1, Kensington, N. S. W. 2033, Australia.

(1) Copyright Australian Mathematical Society 1987, Serial-fee code 0334-2700/87
} 
functions with the instants of switching being preassigned. With this approximation, the original problem is converted into a sequence of optimal parameter selection problems. Each of these optimal parameter selection problems can be viewed as a finite dimensional optimization problem with linear constraints. Thus, they can be solved by any standard quasi-Newton algorithm, such as the one summarized in Appendix IV of [16]. Their optimal solutions will then give rise to suboptimal controls of the original optimal control problem in an obvious manner.

\section{Problem statement}

Consider the following non-linear differential equation

$$
\dot{x}(t)=f(t, x(t), u(t)), t>0
$$

where $x \equiv\left(x_{1}, \ldots, x_{n}\right) \in R^{n}$ and $u \equiv\left(u_{1}, \ldots, u_{r}\right) \in R^{r}$ are, respectively, the state and control vectors, and $f$ is continuously differentiable in all its variables. The initial condition for the differential equation (1a) is

$$
x(0)=x^{0}
$$

where $x^{0}$ is a given vector in $R^{n}$.

Let

$$
U \equiv\left\{v \equiv\left(v_{1}, \ldots, v_{r}\right) \in R^{r}:\left(C^{i}\right)^{T} v \leqslant b_{i}, i=1, \ldots, q\right\},
$$

where $C^{i}, i=1, \ldots, q$, are $r$ vectors, $b_{i}, i=1, \ldots, q$, are real numbers, and the superscript ' $T$ ' denotes transpose.

A bounded measurable function from $[0, \infty) \rightarrow U$ is called an admissible control. Let $\mathscr{U}$ be the class of all such admissible controls.

For each $u \in \mathscr{U}$, let $x(u)$ denote the corresponding solution of the system (1). Let $\Phi: R^{n} \rightarrow R^{1}$ be continuously differentiable, and let $T$ be the first positive time such that

$$
\Phi(x(u)(T))=0
$$

Clearly, the terminating time $T$ depends on $u$ and is a variable. This differs significantly from the well-known case where $T$ is fixed. In the latter case, the end-point lies on a curve given by $\phi(x(u)(T))=0$, so that the boundary condition for the co-state is a "transversality condition"

$$
\psi(T)=-\xi \frac{\partial \phi(x(u)(T))}{\partial x}
$$

for $\xi \geqslant 0$. In the case considered in this paper, the boundary condition for the co-state needs to be derived differently, as the terminating time $T$ is now a variable rather than fixed. The derivation of this boundary condition is also the main theoretical contribution of this paper. 
It is assumed that, for each $u \in \mathscr{U}$, there exists a $T<\infty$ such that condition (2) is satisfied. This assumption is not really a "restriction" as we will see in Section 5, where an example involving the maximization of the range of an aircraft-like projectile is considered.

We may now state the optimal control problem, denoted by $(P)$, formally as:

Subject to the system (1), find a control $u \in \mathscr{U}$ such that the cost functional

$$
J(u)=F(x(u)(T))+\int_{0}^{T} f_{0}(t, x(u)(t), u(t)) d t
$$

is minimized over $\mathscr{U}$, where $T$ is such that condition (2) is satisfied.

For the functions $F$ and $f_{0}$, we assume that the following conditions are satisfied:

1. $F: R^{n} \rightarrow R^{1}$ is continuously differentiable.

2. $f_{0}:[0, \infty) \times R^{n} \times U \rightarrow R^{1}$ is continuously differentiable in all its variables.

\section{A control parametrization algorithm}

In this section, our aim is to devise a computational algorithm for solving the problem $(P)$, by using the control parametrization technique. The method employed is similar to that used in [5], [6], [9], [11]-[13], [15] and [18].

To begin, we will describe the method by which the sequence of optimal parameter selection problems, whose solutions 'converge' to the solution of the optimal control problem $(P)$, is constructed.

Since the process involves choosing parameters on a finite number of intervals, we need to choose some upper bound $T_{\max }$, so that $T^{*}$, where $T^{*}$ corresponds to the optimal control $u^{*}$ and is such that

$$
0<T^{*} \leqslant T_{\max }
$$

In practice, the computational algorithm may require that $T_{\max }$ be considerably larger than $T^{*}$.

Having chosen $T_{\max }$, let $\left\{I^{P}\right\}$ be a sequence of partitions of the interval $\left[0, T_{\max }\right]$, such that $I^{p}$ has $n_{p}$ elements, $I^{p+1}$ is a refinement of $I^{p}$, and $\left\|I^{p}\right\| \rightarrow 0$ as $p \rightarrow \infty$, where $\left\|I^{p}\right\|$ denotes the length of the largest interval in the partition $I^{p}$.

Further, let $D_{p}$ be the class of all those piecewise constant functions from $\left[0, T_{\max }\right]$ into $R^{r}$ which are consistent with the partition $I^{p}$. Let $\sigma^{p}$ be the vector of values which uniquely describes a function $u$ in $D_{p}$, and let $\Sigma_{p}$ be the class of all such vectors.

To restrict the controls in $\mathscr{U} \cap D_{p}$, the control restraints are reduced to:

$$
\left(C^{i}\right)^{T} \sigma_{k}^{p} \leqslant b_{i}, \quad k=1, \ldots, n_{p} ; i=1, \ldots, q,
$$


where

$$
\sigma^{p} \equiv\left(\sigma_{p}^{p}, \ldots, \sigma_{n_{p}}^{p}\right) \in \Sigma_{p} \text { and for each } k=1, \ldots, n_{p}, \sigma_{k}^{p} \text { is a } r \text { vector. }
$$

The problem $(P)$ may now be approximated by the following problem:

Subject to the system

$$
\begin{gathered}
\dot{y}(t)=\tilde{f}\left(t, y(t), \sigma^{p}\right) \\
y(0)=x^{0}
\end{gathered}
$$

find a parameter vector $\sigma^{p} \in \Sigma_{p}$ such that

$$
\tilde{J}\left(\sigma^{p}\right)=F\left(y\left(\sigma^{p}\right)(T)\right)+\int_{0}^{T} \tilde{f}_{0}\left(t, y\left(\sigma^{p}\right)(t), \sigma^{p}\right) d t
$$

is minimized over $\Sigma_{p}$, where $y\left(\sigma^{p}\right)$ denotes the solution of the system (5) corresponding to the parameter vector $\sigma^{p} \in \Sigma_{p}, T$ is the first positive time such that

$$
\Phi\left(y\left(\sigma^{p}\right)(T)\right)=0,
$$

and $\tilde{f}$ and $\tilde{f}_{0}$ are, respectively, constructed from $f$ and $f_{0}$ in an obvious manner. More precisely,

$$
\tilde{f}\left(t, y\left(\sigma^{p}\right)(t), \sigma^{p}\right) \equiv f(t, x(u)(t), u(t))
$$

and

$$
\tilde{f}_{0}\left(t, y\left(\sigma^{p}\right)(t), \sigma^{p}\right) \equiv f_{0}(t, x(u)(t), u(t)),
$$

with

$$
u(t) \equiv \sum_{k=1}^{n_{p}} \sigma_{k} \chi_{I_{k}}(t)
$$

where $\chi_{I_{k}}$ denotes the characteristic function of $I_{k}, I_{k}=\left[t_{k-1}, t_{k}\right), \quad k=$ $1, \ldots, n_{p}, t_{0}=0$ and $t_{n_{p}}=T_{\max }$.

Thus, to solve the optimal control $(P)$, we suggest that one solves the finite dimensional optimization problem $\left(P_{p}\right)$ successively for $p=p_{0}, p_{1}, p_{2}, \ldots$, where $p_{i}, i=0,1, \ldots$, are positive integers such that $p_{i+1}$ is some multiple of $p_{i}$.

In order to devise a computational method for solving the problem $\left(P_{p}\right)$, we will need to derive a formula for the gradient of the cost functional $J$.

To begin, we define the Hamiltonian function $H$ as follows:

$$
H(t, y, \sigma, \psi) \equiv \tilde{f}_{0}(t, y, \sigma)+\tilde{f}(t, y, \sigma) \cdot \psi,
$$

where "." denotes the usual inner product in a Euclidean space.

Now, for each $\sigma^{p} \in \Sigma_{p}$, let us consider the following system

$\dot{\psi}(t)=-\partial / \partial y H\left(t, y\left(\sigma^{p}\right)(t), \sigma^{p}, \psi\left(\sigma^{p}\right)(t)\right), t \in[0, T]$

$\psi(T)=\partial / \partial y F\left(y\left(\sigma^{P}\right)(T)\right)$

$-\frac{\left[\tilde{f}_{0}\left(T, y\left(\sigma^{p}\right)(T), \sigma^{p}\right)+\partial / \partial y F\left(y\left(\sigma^{p}\right)(T)\right) \cdot \tilde{f}\left(T, y\left(\sigma^{p}\right)(T), \sigma^{p}\right)\right]\left[\partial / \partial y \Phi\left(y\left(\sigma^{p}\right)(T)\right)\right]}{\partial / \partial y \Phi\left(y\left(\sigma^{p}\right)(T)\right) \cdot \tilde{f}\left(T, y\left(\sigma^{p}\right)(T), \sigma^{p}\right)}$ 
where $T$ is the first positive time such that condition (7) is satisfied and is assumed to be a continuous point of the functions $\tilde{f}_{0}$ and $\tilde{f}$.

$\partial / \partial y H\left(t, y\left(\sigma^{p}\right)(t), \sigma^{p}, \psi\left(\sigma^{p}\right)(t)\right)=\partial /\left.\partial y H\left(t, y, \sigma^{p}, \psi\left(\sigma^{p}\right)(t)\right)\right|_{y=y\left(\sigma^{p}\right)(t)}$ is the gradient of $H\left(t, y, \sigma^{p}, \psi\left(\sigma^{p}\right)(t)\right)$ with respect to $y$ evaluated at $y=y\left(\sigma^{p}\right)(t)$; and $\partial / \partial y F\left(y\left(\sigma^{p}\right)(T)\right)$ and $\partial / \partial y \Phi\left(y\left(\sigma^{p}\right)(T)\right)$ are to be understood similarly.

THEOREM 1. For each integer $p>0$, consider the problem $\left(P_{p}\right)$. Then, the gradient of the cost functional (6) evaluated at $\sigma^{p}$ is given by

$$
\tilde{J}_{\sigma}\left(\sigma^{p}\right)=\int_{0}^{T} \partial / \partial \sigma H\left(t, y\left(\sigma^{p}\right)(t), \sigma^{p}, \psi\left(\sigma^{p}\right)(t)\right) d t
$$

Proof. Let $\sigma^{p} \in \Sigma_{p}$ be given, and let $\rho^{p} \in R^{n_{p} r}$ be such that $\sigma^{p}+\rho^{p} \in \Sigma_{p}$. Then,

$$
\sigma^{p}(\varepsilon) \equiv \sigma^{p}+\varepsilon \rho^{p}=\varepsilon\left(\sigma^{p}+\rho^{p}\right)+(1-\varepsilon) \sigma^{p} \in \Sigma_{p}
$$

for all $\varepsilon, 0 \leqslant \varepsilon \leqslant 1$.

For brevity, let $y(\cdot)$ and $y(\cdot, \varepsilon)$ denote, respectively, the solutions of the system (5) corresponding to $\sigma^{p}$ and $\sigma^{p}(\varepsilon)$. Furthermore, let $T(\varepsilon)$ be the first positive time such that

$$
\Phi(y(T(\varepsilon), \varepsilon))=0
$$

Clearly,

and

$$
y(t, \varepsilon)=x^{0}+\int_{0}^{t} \tilde{f}\left(\tau, y(\tau, \varepsilon), \sigma^{p}(\varepsilon)\right) d \tau
$$

$$
y(T(\varepsilon), \varepsilon)=x^{0}+\int_{0}^{T(\varepsilon)} \tilde{f}\left(\tau, y(\tau, \varepsilon), \sigma^{p}(\varepsilon)\right) d \tau .
$$

Differentiating each of these expressions with respect to $\varepsilon$, and then letting $\varepsilon \rightarrow 0$ gives

$$
\begin{aligned}
d /\left.d \varepsilon y(t, \varepsilon)\right|_{e=0}=\int_{0}^{t}\{\partial / \partial y \tilde{f}(\tau, y(\tau) & \left., \sigma^{p}\right) \cdot d /\left.d \varepsilon y(\tau, \varepsilon)\right|_{e=0} \\
& \left.+\partial / \partial \sigma \tilde{f}\left(\tau, y(\tau), \sigma^{P}\right) \cdot \rho^{p}\right\} d \tau
\end{aligned}
$$

and

$$
\begin{aligned}
d /\left.d \varepsilon y(T(\varepsilon), \varepsilon)\right|_{e=0}= & \int_{0}^{T}\left\{\partial / \partial y \tilde{f}\left(\tau, y(\tau), \sigma^{p}\right) \cdot d /\left.d \varepsilon y(\tau, \varepsilon)\right|_{\varepsilon=0}\right. \\
& \left.+\partial / \partial \sigma \tilde{f}\left(\tau, y(\tau), \sigma^{p}\right) \cdot \rho^{p}\right\} d \tau \\
& +\tilde{f}\left(T, y(T), \sigma^{P}\right) d /\left.d \varepsilon T(\varepsilon)\right|_{e=0}
\end{aligned}
$$

where we have used $T=\left.T(\varepsilon)\right|_{e-0}$ in the upper limit of integration in the second expression. 
For convenience, let us adopt the following notation.

$$
\begin{gathered}
\tilde{f}(t) \equiv \tilde{f}\left(t, y(t), \sigma^{p}\right) \\
\tilde{f}_{y}(t) \equiv \partial /\left.\partial y f\left(t, y, \sigma^{p}\right)\right|_{y=y(t)} \\
\tilde{f}_{\sigma}(t) \equiv \partial /\left.\partial \sigma \tilde{f}(t, y(t), \sigma)\right|_{\sigma=\sigma^{p}} \\
\delta y(t) \equiv d /\left.d \varepsilon y(t, \varepsilon)\right|_{\varepsilon=0}
\end{gathered}
$$

and

$$
\delta T \equiv d /\left.d \varepsilon T(\varepsilon)\right|_{\varepsilon=0}
$$

Substituting these abbreviations into equations (14) and (15), it follows, respectively, that

$$
\delta y(t)=\int_{0}^{t}\left\{\tilde{f}_{y}(\tau) \cdot \delta y(\tau)+\tilde{f}_{0}(\tau) \cdot \rho^{p}\right\} d \tau
$$

and

$$
\begin{aligned}
d /\left.d \varepsilon y(T(\varepsilon), \varepsilon)\right|_{e=0}= & \int_{0}^{T}\left\{\tilde{f}_{y}(\tau) \cdot \delta y(\tau)+\tilde{f}_{\sigma}(\tau) \cdot \rho^{p}\right\} d \tau \\
& +\tilde{f}\left(T, y(T), \boldsymbol{\sigma}^{p}\right) \delta T \\
= & \delta y(T)+\tilde{f}\left(T, y(T), \sigma^{p}\right) \delta T .
\end{aligned}
$$

Since

$$
\tilde{J}\left(\sigma^{P}(\varepsilon)\right)=F(y(T(\varepsilon), \varepsilon))+\int_{0}^{T(\varepsilon)} \tilde{f}_{0}\left(\tau, y(\tau, \varepsilon), \sigma^{P}(\varepsilon)\right) d \tau,
$$

we have

$$
\begin{aligned}
\tilde{J}\left(\sigma^{p}(\varepsilon)\right)=F(y(T, \varepsilon), \varepsilon)+\int_{0}^{T(\varepsilon)}\{ & H\left(t, y(t, \varepsilon), \sigma^{p}(\varepsilon), \psi(t, \varepsilon)\right) \\
& \left.-\tilde{f}\left(t, y(t, \varepsilon), \sigma^{p}(\varepsilon)\right) \cdot \psi(t, \varepsilon)\right\} d t,
\end{aligned}
$$

where $\psi(\cdot, \varepsilon)$ denotes the solution of the co-state system (9) corresponding to $\sigma^{p}(\varepsilon)$. Hence,

$$
\begin{aligned}
d /\left.d \varepsilon \tilde{J}\left(\sigma^{P}(\varepsilon)\right)\right|_{\varepsilon=0}= & \partial / \partial y F(y(t)) \cdot d /\left.d \varepsilon y(T(\varepsilon), \varepsilon)\right|_{\varepsilon=0} \\
& +\int_{0}^{T}\left\{H_{y}(t) \cdot \delta y(t)+H_{\sigma}(t) \cdot \rho^{P}\right. \\
& \left.+H_{\psi}(t) \cdot \delta \psi(t)\right\} d t+\tilde{f}_{0}\left(T, y(T), \sigma^{p}\right) \delta T \\
& -\int_{0}^{T}\left\{d /\left.d \varepsilon \tilde{f}\left(t, y(t, \varepsilon), \sigma^{p}\right)\right|_{\varepsilon=0} \cdot \psi(t)\right. \\
& \left.+\tilde{f}\left(t, y(t), \sigma^{P}\right) \cdot \delta \psi(t)\right\} d t,
\end{aligned}
$$


where $\psi(\cdot)$ denotes the solution of the co-state system (9) corresponding to $\sigma^{P}$, and where

$$
\begin{aligned}
& H_{y}(t) \equiv \partial /\left.\partial y H\left(t, y, \sigma^{p}, \psi(t)\right)\right|_{y=y(t)}, \\
& H_{\sigma}(t)=\partial /\left.\partial \sigma H(t, y(t), \sigma, \psi(t))\right|_{\sigma=\sigma^{p}}, \\
& H_{\psi}(t)=\partial /\left.\partial \psi H\left(t, y(t), \sigma^{p}, \psi\right)\right|_{\psi=\psi(t)},
\end{aligned}
$$

and

$$
\delta \psi(t)=d /\left.d \varepsilon \psi(t, \varepsilon)\right|_{\varepsilon=0} .
$$

Again for convenience, let

$$
\begin{gathered}
\delta \tilde{J}\left(\sigma^{p}\right)=d /\left.d \varepsilon \tilde{J}\left(\sigma^{p}(\varepsilon)\right)\right|_{e=0}, \\
F_{y}(y(T))=\partial /\left.\partial y F(y)\right|_{y=y(T)}, \\
\tilde{f}(t, \varepsilon)=\tilde{f}\left(t, y(t, \varepsilon), \sigma^{P}(\varepsilon)\right), \\
\tilde{f}(t)=\tilde{f}\left(t, y(t), \sigma^{p}\right), \\
\tilde{f}_{0}(t, \varepsilon)=f_{0}\left(t, y(t, \varepsilon), \sigma^{p}(\varepsilon)\right),
\end{gathered}
$$

and

$$
\tilde{f}_{0}(t)=\tilde{f}_{0}\left(t, y(t), \sigma^{p}\right) .
$$

Then, by (17), equation (19) can be written as

$$
\begin{aligned}
\delta \tilde{J}\left(\sigma^{P}\right)= & F_{y}(y(T)) \cdot(\delta y(T)+\tilde{f}(T) \delta T) \\
& +\int_{0}^{T}\left\{H_{y}(t) \cdot \delta y(t)+H_{\sigma}(t) \cdot \rho^{p}+H_{\psi}(t) \cdot \delta \psi(t)\right\} d t \\
& -\int_{0}^{T}\left\{d /\left.d \varepsilon \tilde{f}(t, \varepsilon)\right|_{\varepsilon=0} \cdot \psi(t)+\tilde{f}(t) \cdot \delta \psi(t)\right\} d t+\tilde{f}_{0}(T) \delta T .
\end{aligned}
$$

Now, we note that

$$
\dot{\psi}(t)=-H_{y}(t)
$$

and

$$
\dot{y}(t)=H_{\psi}(t)
$$

Thus, (20) is reduced to

$$
\begin{aligned}
\delta \tilde{J}\left(\sigma^{p}\right)= & F_{y}(y(T)) \cdot(\delta y(T)+\tilde{f}(T) \delta T) \\
& +\int_{0}^{T}\left\{-\dot{\psi}(t) \cdot \delta y(t)+H_{0}(t) \cdot \rho^{p}+\tilde{f}(t) \cdot \delta \psi(t)\right\} d t \\
& -\int_{0}^{T}\left\{d /\left.d \varepsilon f(t, \varepsilon)\right|_{e=0} \cdot \psi(t)+\tilde{f}(t) \cdot \delta \psi(t)\right\} d t+\tilde{f}_{0}(T) \delta T \\
= & F_{y}(y(T)) \cdot(\delta y(T)+\tilde{f}(T) \delta d T) \\
& +\int_{0}^{T}\left\{H_{0}(t) \cdot \rho^{p}-\left[\dot{\psi}(t) \cdot \delta y(t)+d /\left.d \varepsilon \tilde{f}(t, \varepsilon)\right|_{\varepsilon=0} \cdot \psi(t)\right]\right\} d t \\
& +\tilde{f}_{0}(T) \delta T .
\end{aligned}
$$


However,

$$
d /\left.d \varepsilon \tilde{f}(t, \varepsilon)\right|_{\varepsilon=0}=\tilde{f}_{y}(t) \cdot \delta y(t)+\tilde{f}_{\sigma}(t) \cdot \rho^{p}
$$

and, from (16), we have

$$
\delta \dot{y}(t)=\tilde{f}_{y}(t) \cdot \delta y(t)+\tilde{f}_{0}(t) \cdot \rho^{p} .
$$

Thus, it follows from (20) that

$$
\begin{aligned}
\delta \tilde{J}\left(\sigma^{p}\right)= & F_{y}(y(T)) \cdot(\delta y(T)+\tilde{f}(T) \delta T) \\
& +\int_{0}^{T}\left\{H_{\sigma}(t) \cdot \rho^{p}-d / d t(\psi(t) \cdot \delta y(t))\right\} d t \\
& +\tilde{f}_{0}(T) \delta T \\
= & F_{y}(y(T)) \cdot(\delta y(T)+\tilde{f}(T) \delta T) \\
& +\int_{0}^{T} H_{\sigma}(t) \cdot \rho^{p} d t-[\psi(t) \cdot \delta y(t)]_{0}^{T}+\tilde{f}_{0}(T) \delta T .
\end{aligned}
$$

However,

$$
y(0)=y(0, \varepsilon)=x^{0}
$$

implies that

$$
\delta y(0)=0
$$

Hence,

$$
\begin{aligned}
\delta \tilde{J}\left(\sigma^{p}\right)= & F_{y}(y(T)) \cdot(\delta y(T)+\tilde{f}(T) \delta T) \\
& +\int_{0}^{T} H_{\sigma}(t) \cdot \rho^{p} d t-\psi(T) \cdot \delta y(T)+\tilde{f}_{0}(T) \delta T .
\end{aligned}
$$

Now, let us consider the constraint:

$$
\Phi(y(T, \varepsilon))=0 .
$$

Differentiating with respect to $\varepsilon$, and then letting $\varepsilon \rightarrow 0$ gives:

$$
\Phi_{y}(y(T)) \cdot d /\left.d \varepsilon y(T(\varepsilon), \varepsilon)\right|_{e=0}=0,
$$

where $\Phi_{y}(y(T))=\partial /\left.\partial y \Phi(y)\right|_{y=y(T)}$.

Combining (26) and (17), we have

$$
\begin{aligned}
0 & =\Phi_{y}(y(T)) \cdot(\delta y(T)+\tilde{f}(T) \delta T) \\
& =\Phi_{y}(y(T)) \cdot \delta y(T)+\Phi_{y}(y(T)) \cdot \tilde{f}(T) \delta T .
\end{aligned}
$$

Hence,

$$
\delta T=-\frac{\Phi_{y}(y(T)) \cdot \delta y(T)}{\Phi_{y}(y(T)) \cdot \tilde{f}(T)}
$$


Substituting (27) into (25), we obtain

$$
\begin{aligned}
\delta \tilde{J}\left(\sigma^{p}\right)= & F_{y}(y(T)) \cdot \delta y(T) \\
& -\left[F_{y}(y(T)) \cdot \tilde{f}(T)-\tilde{f}_{0}(T)\right] \frac{\Phi_{y}(y(T)) \cdot \delta y(T)}{\Phi_{y}(y(T)) \cdot \tilde{f}(T)} \\
& +\int_{0}^{T} H_{\sigma}(t) \cdot \rho^{p} d t-\psi(T) \cdot \delta y(T) .
\end{aligned}
$$

Now, by virtue of $(9 b)$, it is clear that

$$
\delta \tilde{J}\left(\sigma^{p}\right)=\int_{0}^{T} H_{\sigma}(t) \cdot \rho^{p} d t .
$$

Since

$$
\delta \tilde{J}\left(\sigma^{p}\right)=d /\left.d \varepsilon \tilde{J}\left(\sigma^{p}(\varepsilon)\right)\right|_{\varepsilon=0}=\tilde{J}_{\sigma}\left(\sigma^{p}\right) \cdot \rho^{p}
$$

and since $\rho^{p} \in R^{n_{p} r}$ is such that

$$
\sigma^{p}+\rho^{p} \in \Sigma_{p},
$$

we conclude that

$$
\tilde{J}_{\sigma}\left(\sigma^{p}\right)=\int_{0}^{T} H_{\sigma}(t) d t .
$$

Thus, the proof is complete.

We are now in a position to construct an algorithm for solving the problem $\left(P_{p}\right)$ as follows:

1. Set $k=0$ and select an initial guess $\sigma^{p, k} \in \Sigma_{p}$.

2. Integrate the system (5) with $\sigma^{p}=\sigma^{p, k}$ forward in time from 0 until $T_{k}$ such that $\mid \Phi\left(y\left(\sigma^{p, k}\right)\left(T_{k}\right) \mid<\varepsilon_{\Phi}\right.$, where $\varepsilon_{\Phi}$ is a preset tolerance, and then integrate the co-state system (9) with $\sigma^{p}=\sigma^{p, k}$ backward in time from $T_{k}$ to 0 .

3. If $t_{j}<T_{k}<t_{j+1}$ (i.e., $T_{k}$ lies in the $j$ th decision interval), then set

$$
\partial / \partial \sigma_{i} \tilde{J}\left(\sigma^{p, k}\right)= \begin{cases}0, & i>j+1 \\ \int_{t_{j}}^{T_{k}} \partial / \partial \sigma_{i} H\left(\sigma^{p, k}\right)\left(t, \sigma^{p, k}\right) d t, & i=j+1 \\ \int_{t_{i+1}}^{t_{t}} \partial / \partial \sigma_{i} H\left(\sigma^{p, k}\right)\left(t, \sigma^{p, k}\right) d t, & i<j+1\end{cases}
$$

where

$$
\begin{gathered}
\sigma^{p, k}=\left(\sigma_{P}^{p, k}, \ldots, \sigma_{n_{p}}^{p, k}\right), \quad \sigma_{i}^{p, k}=\left(\sigma_{i, 1}^{p, k}, \ldots, \sigma_{i, r}^{p, k}\right), \quad i=1, \ldots, r, \\
\partial / \partial \sigma H\left(\sigma^{p, k}\right)\left(t, \sigma^{p, k}\right)=\partial /\left.\partial \sigma H\left(t, y\left(\sigma^{p, k}\right)(t), \sigma, \psi\left(\sigma^{p, k}\right)(t)\right)\right|_{\sigma=\sigma^{p, k}}
\end{gathered}
$$

and $\partial / \partial \sigma_{t} H\left(\sigma^{p, k}\right)\left(t, \sigma^{p, k}\right)$ denotes the $i$ th vector of $\partial / \partial \sigma H\left(\sigma^{p, k}\right)\left(t, \sigma^{p, k}\right)$

4. Solve for $\hat{\sigma}^{p, k} \in \Sigma_{p}$ so that

$$
\tilde{J}_{\sigma}\left(\sigma^{p, k}\right) \cdot \hat{\sigma}^{p, k}=\min _{\sigma^{p} \in \Sigma_{p}} \tilde{J}_{\sigma}\left(\sigma^{p, k}\right) \cdot \sigma^{p},
$$


where

$$
\tilde{J}_{\mathrm{o}}\left(\sigma^{p, k}\right)=\left(\partial / \partial \sigma_{1} \tilde{J}\left(\sigma^{p, k}\right), \ldots, \partial / \partial \sigma_{n_{p}} \tilde{J}\left(\sigma^{p, k}\right)\right) .
$$

5. Solve for $\lambda_{p, k}>0$ (by a line search) so that

$$
\begin{array}{r}
\tilde{J}\left(\sigma^{p, k}+\lambda_{p, k}\left(\hat{\sigma}^{p, k}-\sigma^{p, k}\right)\right)=\min \left\{\tilde{J}\left(\sigma^{p, k}+\lambda_{p}\left(\hat{\sigma}^{p, k}-\sigma^{p, k}\right)\right): \lambda_{p} \geqslant 0\right. \\
\text { and } \left.\sigma^{p, k}+\lambda_{p}\left(\hat{\sigma}^{p, k}-\sigma^{p, k}\right) \in \Sigma_{p}\right\}
\end{array}
$$

6. Set $\sigma^{p, k+1}=\sigma^{p, k}+\lambda_{p, k}\left(\hat{\sigma}^{p, k}-\sigma^{p, k}\right)$.

7. Stop if $\lambda_{p, k}=0$; otherwise go back to Step 2 with $\sigma=\sigma^{p, k}$.

Practical considerations require us to terminate the algorithm if any of the following conditions is satisfied.

(a) If while integrating the system forward $t=T_{\max }$ and $\mid \Phi\left(y\left(\sigma^{p, k}\right)(t) \mid>\varepsilon_{\Phi}\right.$ then stop, as we need a larger $T_{\max }$. Restart the problem with the control obtained just prior to termination as the initial guess.

(b) $\left|\tilde{J}\left(\sigma^{p, k+1}\right)-\tilde{J}\left(\sigma^{p, k}\right)\right|<\varepsilon_{J}$

(c) $\lambda_{p, k}<\varepsilon_{\lambda}$.

Remark. Recall that the terminating time $T$ in Theorem 1 is assumed to be a continuous point of the functions $\tilde{f}_{0}$ and $\tilde{f}$, and that $\sigma^{p, k} \in \Sigma_{p}$ determines uniquely a control

$$
u^{p, k}(t)=\sum_{l=1}^{n_{p}} \sigma \rho^{p, k} \chi_{I_{l}}(t),
$$

where $I_{l}=\left[t_{t-1}, t_{l}\right)$. Now, in Step 2 of the algorithm, $T_{k}$ is the terminating time. Thus, it is clear that $T_{k}$ is a continuous point of the functions $\tilde{f}_{0}$ and $\tilde{f}$ only if $T_{k} \neq t_{L}$ for all $L \in\left\{1, \ldots, n_{p}\right\}$. If there exists an $L \in\left\{1, \ldots, n_{p}\right\}$ such that $T_{k}=t_{L}$, then $T_{k}$ is no longer a continuous point of the functions $\tilde{f}_{0}$ and $\tilde{f}$. Thus, we need to alter the definition of $u^{p, k}$ as follows:

$$
u^{p, k}(t)=\sum_{l=1}^{n_{p}} \tilde{\sigma} \rho^{p, k} \chi_{l}(t)
$$

where

$$
\tilde{\boldsymbol{\sigma}} p^{, k}=\sigma f^{, k} \text { for all } l \neq L+1
$$

and

$$
\tilde{\sigma} p^{, k}=\sigma \ell^{p, k} \text { for } l=L+1
$$


Then, we write $\tilde{\sigma}^{p, k}$ as $\sigma^{p, k}$ and go to Step 3 . Here, it is not necessary to go back to Step 2, as the corresponding result of Step 2 would remain the same as before. With this modification, $T_{k}$ is now a continuous point of the functions $\tilde{f}$ and $\tilde{f}_{0}$, also. In practice, it is, however, most unlikely that there would exist an $L \in$ $\left\{1, \ldots, n_{p}\right\}$ such that $T_{k}=t_{L}$.

\section{COMMENTS ON STOPPING CRITERIA.}

i. $\varepsilon_{\Phi}>0$ is the required tolerance in $\Phi$. In numerically integrating the system forward we will often integrate through a zero of $\Phi$ with a large integration step. In practice, we test for a sign change in $\Phi$ after each integration step. If a sign change occurs in $\Phi$ but $\partial \Phi\left(y\left(\sigma^{p, k}\right)(t)\right) \mid>\varepsilon_{\Phi}$, we repeat the previous integration step with half the previous step size. This process is repeated until the required tolerance is obtained.

ii. $\varepsilon_{J}>0$ is a sufficiently small decrease in the cost functional for the optimization routine to claim a minimum.

iii. $\varepsilon_{\lambda}>0$ is a sufficiently small step size for the optimization routine to claim a minimum.

\section{Implementation of the algorithm in a FORTRAN program}

For fixed time optimal control problems, a corresponding algorithm may be found in [11], [12], and [15]. This algorithm has already been implemented by the third author at the School of Mathematics, University of New South Wales, Australia, as a FORTRAN program, CONOPT. This FORTRAN program has been modified by the fourth author to cover the problem under discussion by using the algorithm developed in the previous section. The details of the implementation are not relevant to this paper. We shall, however, remark that the optimization is carried out using a linearly constrained quasi-Newton method, originally developed by Fletcher in [4], as implemented in the Harwell subroutine library with minor modifications. A summary of this method may be found in Appendix IV of [16].

\section{An example: an aircraft-like projectile}

In this section, we shall consider a model which describes a gliding projectile. This projectile could be launched from an aircraft flying near sea level, with the aircraft being placed in a steep climb just prior to the launch of the projectile, or 


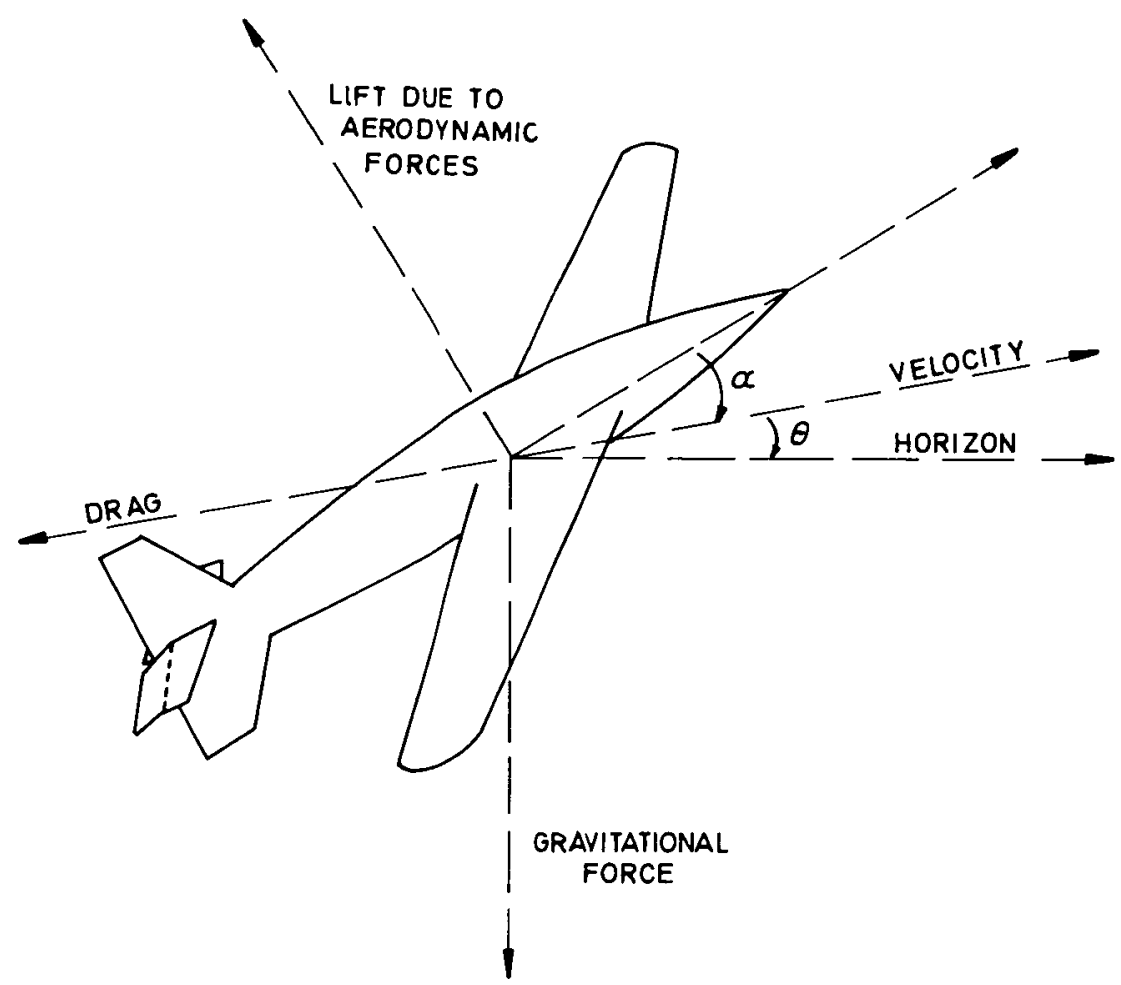

Figure 1. Model of an aircraft-like projectile.

the projectile could be launched from a launching pad. The aim is to launch the projectile at a point as far as possible from the target.

The model has 4 state variables $X(t)=\left(x_{1}(t), x_{2}(t), x_{3}(t), x_{4}(t)\right)$ and one control variable, $u(t)$. It is shown in Figure 1 . The state variables represent the following quantities.

$x_{1}(t) \equiv x=$ horizontal distance from launch point

$x_{2}(t) \equiv h=$ vertical distance above launch point

$x_{3}(t) \equiv v=$ speed of the projectile

$x_{4}(t) \equiv \theta=$ the angle between the velocity vector and the horizon.

The control variable is

$u(t) \equiv \alpha(t)=$ the angle of attack of the projectile.

The mathematical description of this model is:

$$
\begin{aligned}
& \dot{x}=v \cos \theta \\
& \dot{h}=v \sin \theta
\end{aligned}
$$




$$
\begin{gathered}
\dot{v}=-\left(k_{D_{0}}+k_{D_{2}} \alpha^{2}\right) v^{2}-g \sin \theta \\
\dot{\theta}=k_{L} \alpha v-\frac{g}{v} \cos \theta
\end{gathered}
$$

with the fixed initial conditions

$$
X(0)=(0,0,370.0,1.5)
$$

where

$$
\begin{aligned}
& k_{D_{0}}=3.289 \times 10^{-5} \mathrm{~m}^{-1}, \\
& k_{D_{2}}=1.133 \times 10^{-3} \mathrm{~m}^{-1}, \\
& k_{L}=3.289 \times 10^{-3} \mathrm{~m}^{-1} .
\end{aligned}
$$

The problem is then to find a control $u \in \mathscr{U}$ which maximizes the cost functional

$$
J(u)=x(T)
$$

subject to the dynamical system (30), where $T$ is the first positive time such that

$$
h(T)=0
$$

and $U$ is the class of admissible controls which consists of all bounded measurable functions $u:[0, \infty) \rightarrow R^{1}$. In this problem, the terminating criterion is defined by (32).

Since the problem is highly nonlinear and very nonconvex, we can only expect to obtain a local, rather than global, minimum. Consequently, our algorithm should produce "better" local minimum if a good estimate of initial $u(t)$ is entered. For this reason, we shall first use a calculus of variations approach (cf. [3]) to obtain an estimation of some asymptotic results of the problem.

Let us consider the following related problem.

Subject to the dynamical system (30), find a control $u \in L_{2}$ so that the cost functional

$$
J(u)=\int_{0}^{T} \dot{x}(t) d t
$$

is maximized over $L_{2}$.

Incorporating the state constraints using Lagrange's method of undetermined multipliers, the problem becomes to maximize

$$
J(u)=\int_{0}^{T} G(t, x(t), h(t), v(t), \theta(t), \dot{x}(t), \dot{h}(t), \dot{v}(t), \dot{\theta}(t)) d t,
$$


where

$$
\begin{aligned}
G= & \dot{x}(t)+\lambda_{v}(t)\left(\dot{v}+\left(k_{D_{0}}+k_{D_{2}} \alpha^{2}\right) v^{2}+g \sin \theta\right) \\
& +\lambda_{\theta}(t)\left(\dot{\theta}-k_{L} \alpha v+\frac{g}{v} \cos \theta\right) \\
& +\lambda_{H}(t)(\dot{h}-v \sin \theta)+\lambda_{x}(t)(\dot{x}-v \cos \theta) .
\end{aligned}
$$

Substituting this in the Euler equations and considering the component of $G$ which does not include the 'dot' terms $\dot{x}, \dot{h}, \dot{v}, \dot{\theta}$ as $H$ gives the following equations

$$
\begin{gathered}
\dot{x}=v \cos \theta, \\
\dot{h}=v \sin \theta, \\
\dot{v}=-v^{2}\left(k_{D_{0}}+k_{D_{2}} \alpha^{2}\right)-g \sin \theta, \\
\dot{\theta}=k_{L} \alpha v-\frac{g}{v} \cos \theta, \\
\dot{\lambda}_{h}=0 \\
\dot{\lambda}_{v}=2 \lambda_{v} k_{D_{0}} v-\lambda_{\theta} \frac{g}{v^{2}} \cos \theta-\lambda_{h} \sin \theta+\cos \theta, \\
\dot{\lambda}_{\theta}=\lambda_{v} g \cos \theta-\lambda_{\theta} \frac{g}{v} \sin \theta-\lambda_{h} v \cos \theta-v \sin \theta, \\
H=-v \cos \theta+\left(\frac{1}{4} k_{L}^{2} / K_{D_{2}}\right) \lambda_{\theta}^{2} / \lambda_{v} \\
-\lambda_{v}\left(k_{D_{0}} v^{2}+g \sin \theta\right)-\lambda_{\theta} \frac{g}{v} \cos \theta+\lambda_{h} v \sin \theta \\
=\operatorname{constant.}
\end{gathered}
$$

These equations, which are the necessary conditions for the functional (34) to have a maximum (or a minimum), can be solved if all the initial conditions are known. However this is not the case. Although the initial conditions for the trajectory, $X(0)$, are known; we do not know the initial conditions for the Lagrange multipliers, $\theta(0)$. Nonetheless, some asymptotic results are available if we assume that impact occurs when the time is large enough for these asymptotic results to be closely approached.

Assume $\dot{v}, \dot{\theta}, \dot{\alpha}, \dot{\lambda}_{h} \rightarrow 0$ for large $t$. Also, for optimal solutions with 'free' final boundary conditions on $v$ and $\theta$ we have $\lambda_{v}=\lambda_{\theta}=0$ for the final value of $t$. 
The asymptotic results obtained are:

$$
\begin{aligned}
v_{f}^{2} & \rightarrow g \cos \theta_{f} /\left(k_{L} \sqrt{k_{D_{0}} / k_{D_{2}}}\right), \\
\alpha_{f} & \rightarrow \sqrt{k_{D_{0}} / k_{D_{2}}}, \\
\tan \theta_{f} & \rightarrow-2 \sqrt{k_{D_{0}} k_{D_{2}} / k_{L}}, \\
\lambda_{h f} & \rightarrow 0 \\
H_{f} & \rightarrow-v_{f} \cos \theta_{f} .
\end{aligned}
$$

These results indicate that $\alpha \rightarrow 0.17$ asymptotically. We will use this result in the numerical solution of the maximum range problem.

In the statement of the problem in Section 2, we assume that for each $u \in \mathscr{U}$, there exists a $T<\infty$ such that $\Phi(X(T))=0$. We mention that this is not necessarily a "restriction". In reference to the problem stated in this section, we see that it is certainly not a restriction. In this particular case, $\Phi(X(T)) \equiv h(T)$, and no matter what control we should choose, the physics of the model ensures that at some time the projectile will strike the ground, and the condition $\Phi(X(T))=0$ is satisfied.

Since the algorithm is designed to find minima of the cost functional, while we desire to find the maximum range of the projectile, we implement the equivalent problem of minimising the negative of the final range.

The problem, which is to be solved, uses

$$
\begin{aligned}
f_{0} & \equiv 0 \\
F(x(t)) & =-x_{1}(T) \equiv-x(t),(- \text { the final range })
\end{aligned}
$$

in the cost functional.

We proceed to select $T$ as 400 seconds and recall that the asymptotic behavior of the projectile should include a constant control of 0.17 .

With this information, we make an initial estimate of the control as

$$
u(t)= \begin{cases}0 & \text { for } 0 \leqslant t \leqslant 40 \\ 0.17 & \text { for } 40<t \leqslant 400\end{cases}
$$

where $t=40$ seconds is approximately the time at which the projectile reaches the peak of its trajectory if zero control is used throughout the whole time. More precisely, if zero control is used throughout the whole time, the projectile reaches the peak of its trajectory at approximately $t=38$ seconds, where the nearest switching point occurs at $t=40$ seconds, and hence 40 seconds instead of 38 seconds is chosen as the switching time. The application of the algorithm to this initial estimate produces a range of $43302 \mathrm{~m}$. Let the corresponding trajectory and control be referred to as trajectory 1 and control 1, respectively. 


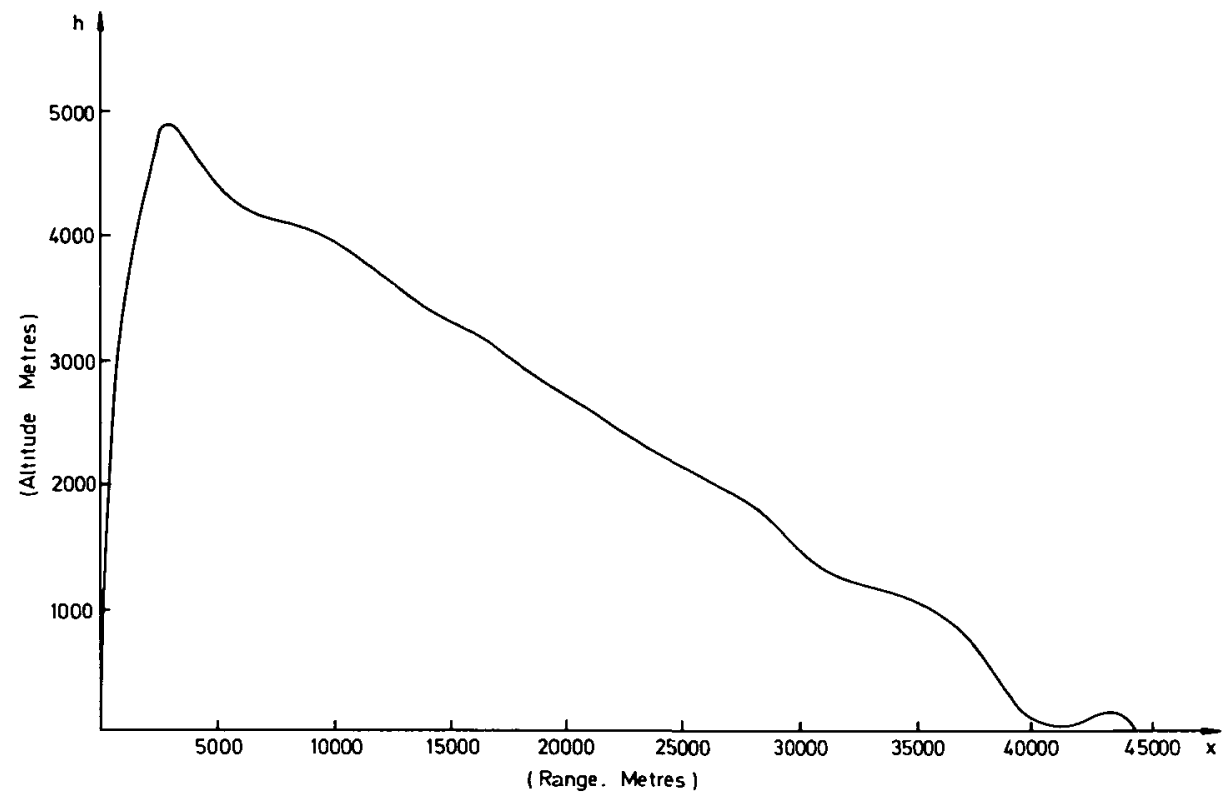

Figure 2(a). Trajectory 3: Range $44710 \mathrm{~m}$.

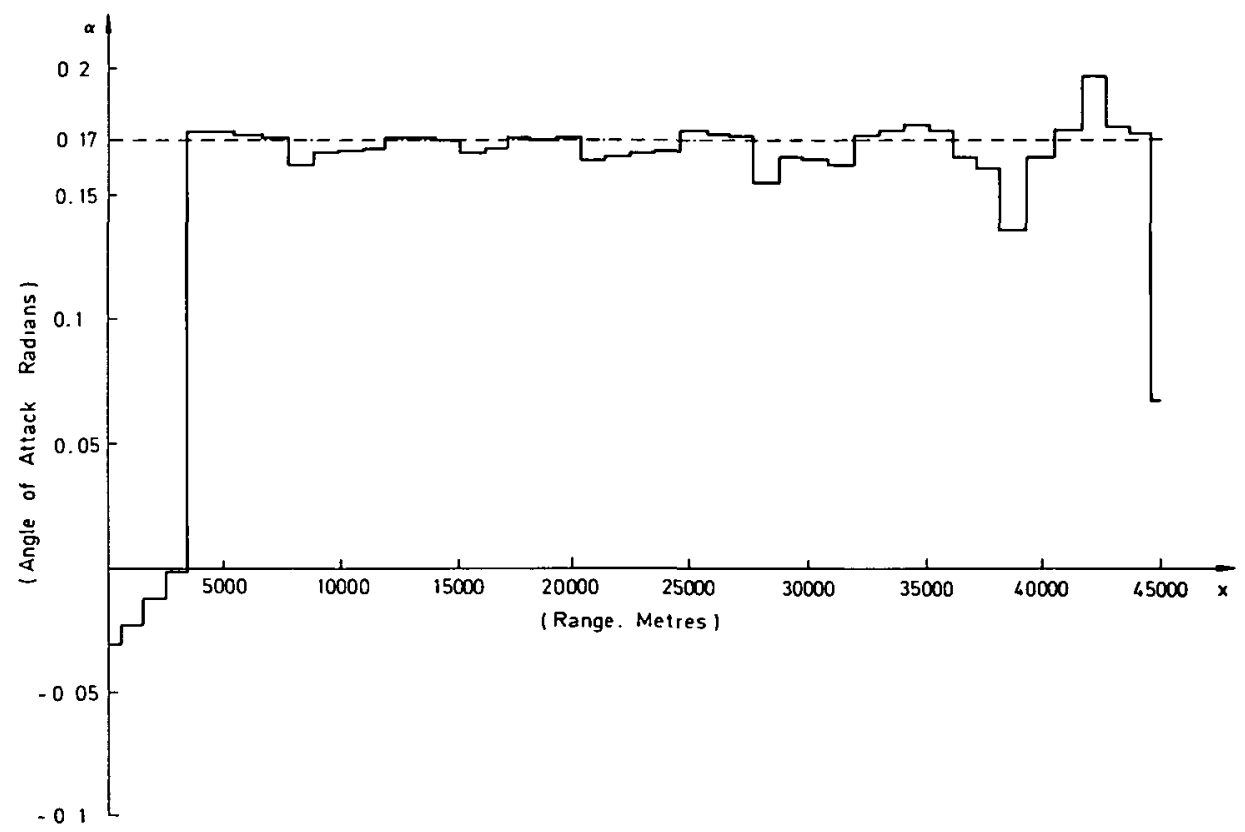

Figure 2(b). Control 3: Range $=44710 \mathrm{~m}$. 
Beginning with this control we obtain an improved range of $44151 \mathrm{~m}$. The trajectory and control so obtained are to be referred to as trajectory 2 and control 2 , respectively. The final dip reaches a minimum height of $0.500 \mathrm{~m}$ at a range of $40.7 \mathrm{~km}$ before recovering and proceeding to climb to subsequent peak of $90 \mathrm{~m}$.

Using the initial portion of the control 2 until the time when the peak of the trajectory 2 is reached and then a subsequent control of 0.17 , the algorithm is applied again. The control and trajectory produced are included in Figure 2 as control 3 and trajectory 3 .

We proceed to modify the program providing the implementation to incorporate the facility to vary the length of the decision intervals over the initial (climbing) phase of the projectile's path.

The subsequent applications of the algorithm use 1 second decision intervals for the first $\mathbf{4 0}$ seconds and 8 seconds decision intervals, as previously, for later times. Since we have a good analytic asessment of the final behaviour we now concentrate our attention on the initial phase of the projectile's path.

The initial estimate supplied for the modified algorithm is control 3. The trajectory and control which are produced from this by applying the modified algorithm are included as trajectory 4 and control 4 in Figure 3, where the range is $45490 \mathrm{~m}$.

In an attempt to determine whether or not a constant glide path gave a superior performance, we now re-configure the problem so that the control is fixed at 0.17 for times greater than 80 seconds. A switching time of 80 seconds is chosen because when a control of 0.17 is used from the peak of the trajectory onwards (trajectory 1 and control 1) the oscillations in the glide path have smoothed out after an elapsed time of 80 seconds.

The initial estimate used is zero for the first 40 seconds, and then a constant control of 0.17 . The resulting optimal solution (trajectory 5 and control 5 in Figure 4) gives a smooth control in the climbing phase of the trajectory, which is as hoped for, but a range of only $43702 \mathrm{~m}$. This is also a good example of the insensitivity of the range to the control.

In a search for an easily implemented control, we proceed to cut off the peak in the control near 0.13 and reduce all values above 0.17 to 0.17 to produce control 6. The corresponding trajectory (trajectory 6) has a final range of $43666 \mathrm{~m}$, a loss of only $336 \mathrm{~m}$.

An examination of the controls produced by the algorithm up to this point in relation to the corresponding maximum ranges would seem to indicate that the model is insensitive to the control, in that a wide range of possible controls produce comparable final ranges. This is in fact what one would expect, since any model which was subject to large changes in the final range due to small changes in the control would represent a very unstable physical system. 


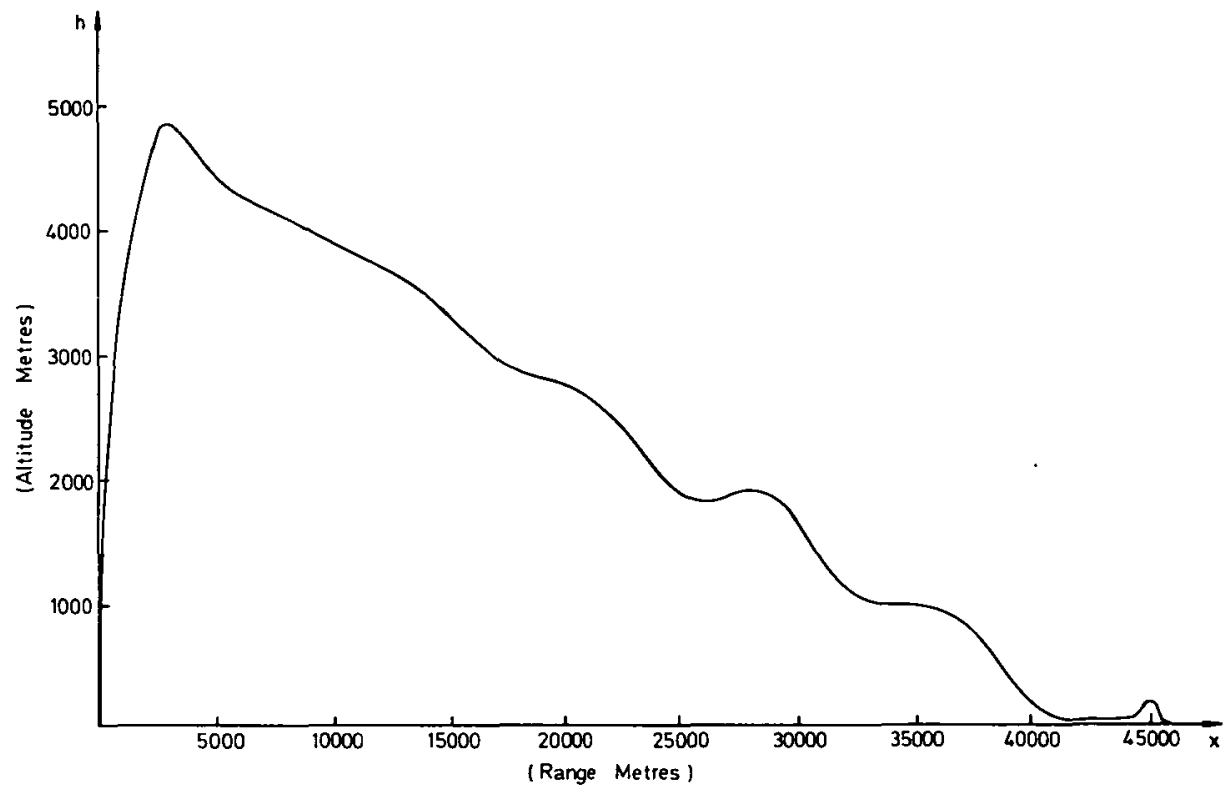

Figure 3(a). Trajectory 4: Range $=45490 \mathrm{~m}$.

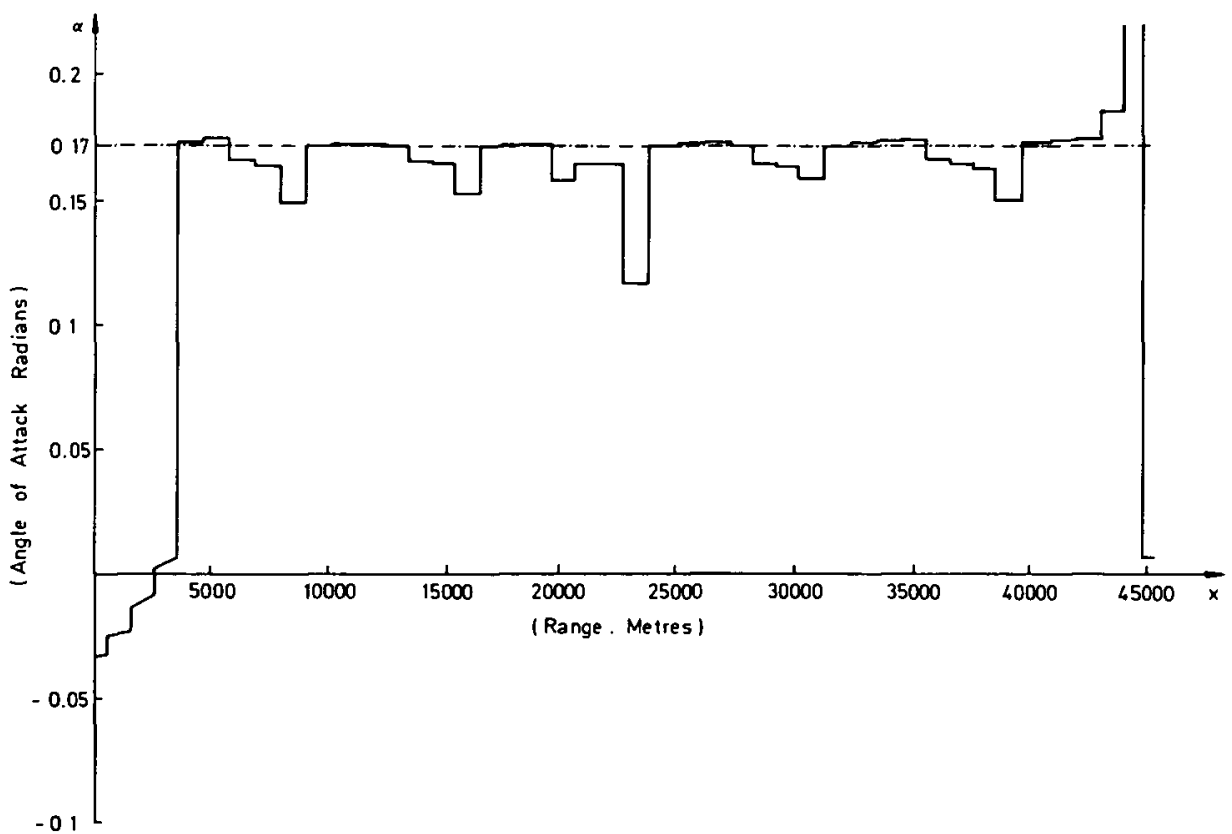

Figure 3(b). Control 4: Range $=45490 \mathrm{~m}$. 


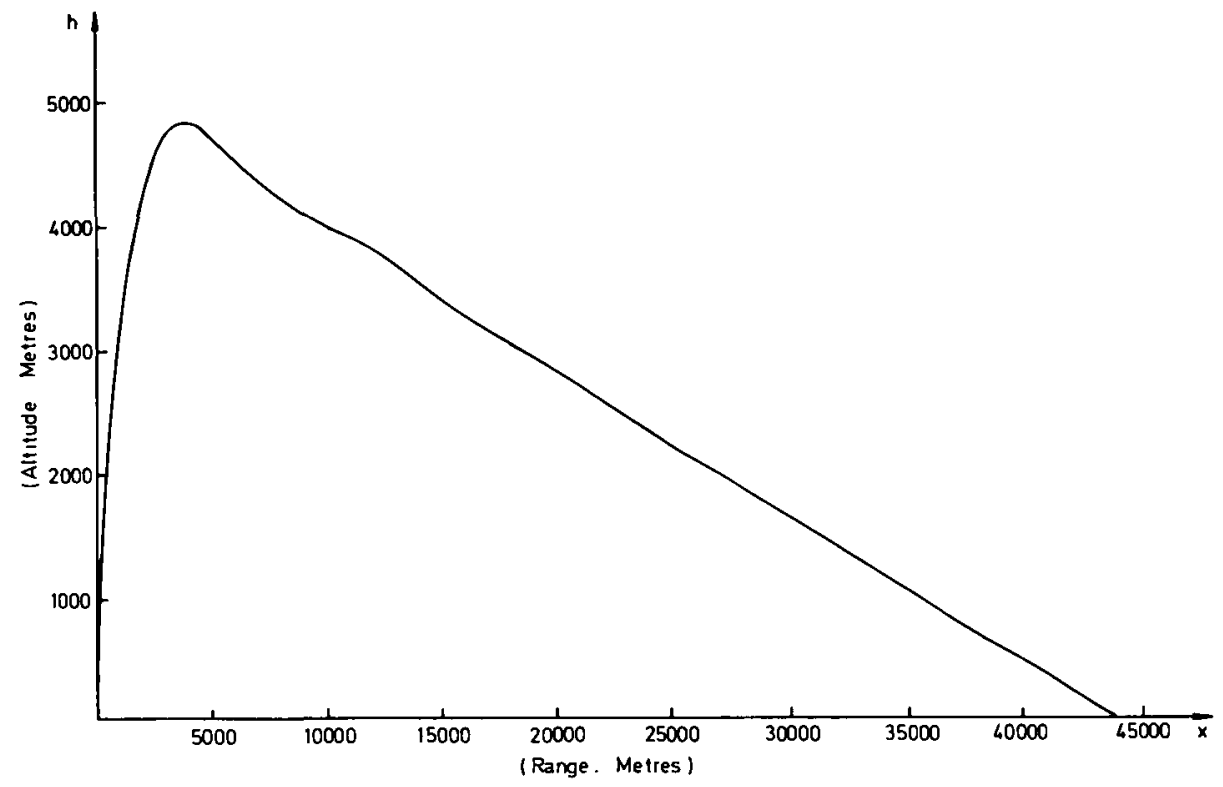

Figure 4(a). Trajectory 5: Range $=43702 \mathrm{~m}$.

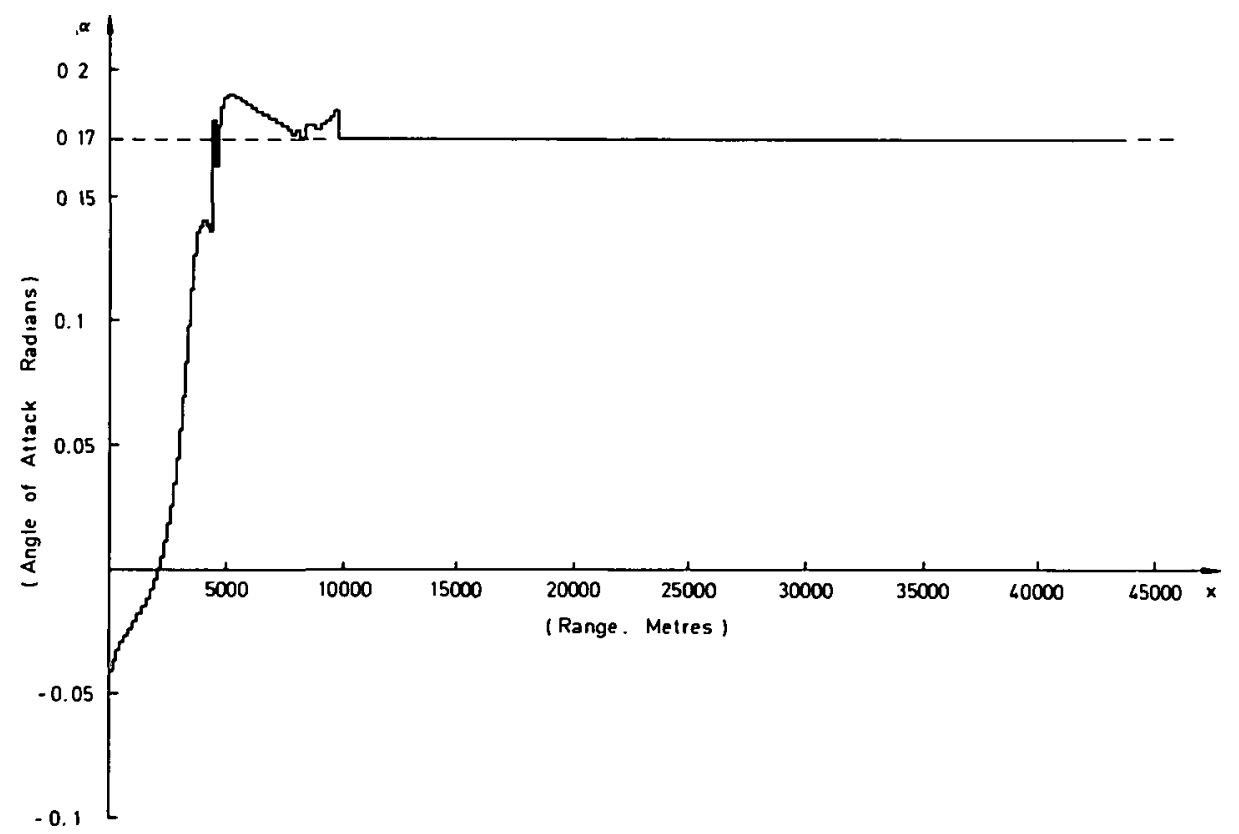

Figure 4(b). Control 5: Range $=43702 \mathrm{~m}$. 


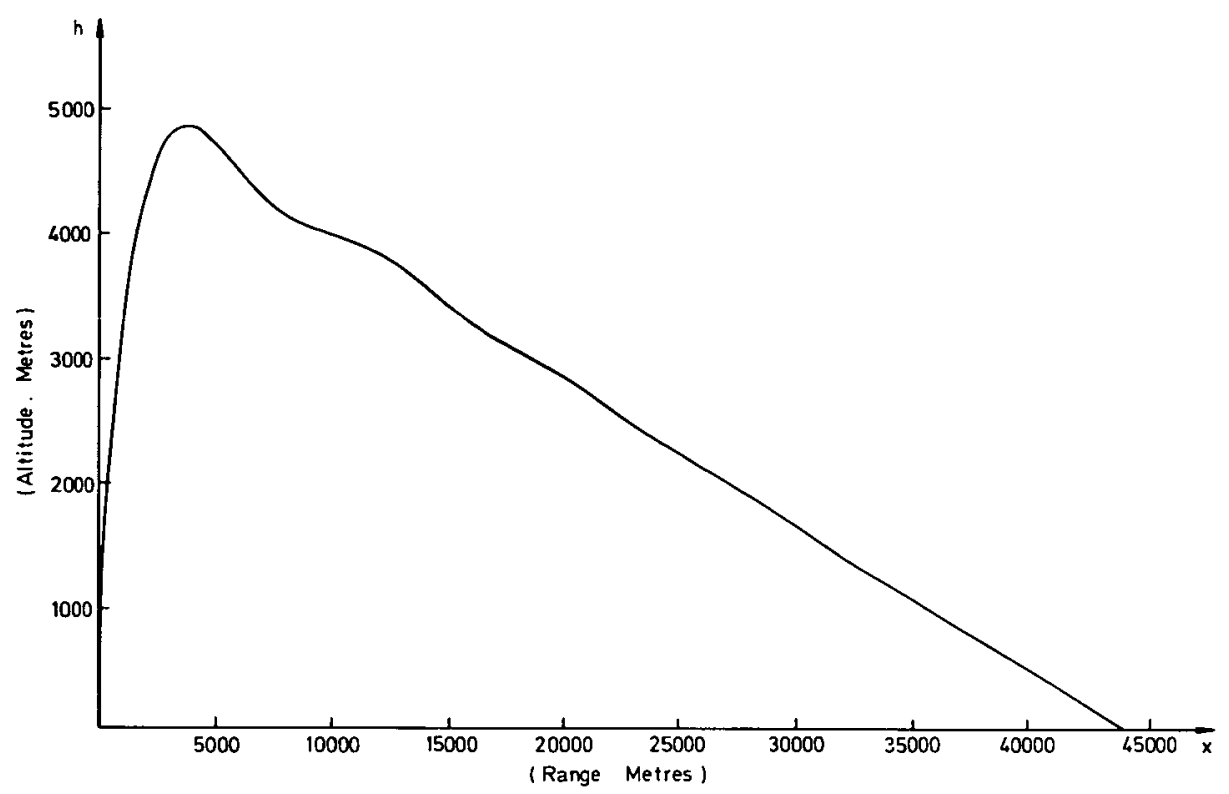

Figure 5(a). Trajectory 6: Range $=43666 \mathrm{~m}$.

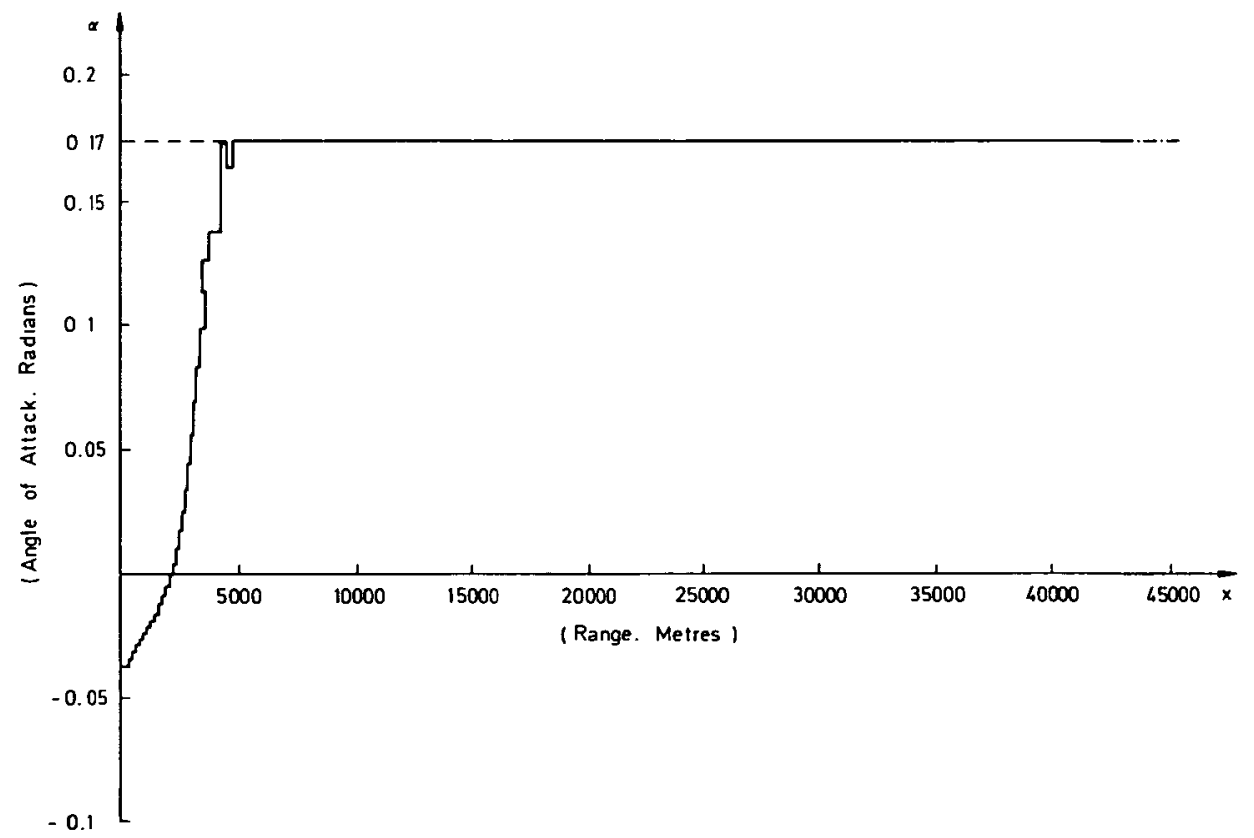

Figure 5(b). Control 6: Range $=43666 \mathrm{~m}$. 


\section{References}

[1] W. Balston, Mathematical Methods for Digital Computers (Wiley, 1960) 110-120.

[2] W. E. Bosarge Jr., and O. G. Johnson, "Direct Method Approximation to the State Regulator Control Problem Using a Ritz-Trefftz Suboptimal Control", IEEE Trans. Automat. Control AC-15 (1970), 627-631.

[3] L. E. Elsgolc, Calculus of Variations (Pergamon Press, London, 1962).

[4] R. Fletcher, "A General Quadratic Programming Algorithm", J. Inst. Math. Applic. 7 (1971), 76-91.

[5] L. Hausdorff, Gradient Optimizatıon and Nonlinear Control (Wiley, New York, 1976).

[6] G. A. Hicks and W. H. Ray, "Approximation Methods for Optimal Control Synthesis", Canad. J. Chem. Eng. 49 (1971), 522-528.

[7] E. Polak, Computational Methods in Optimization (Academic Press, New York, 1971).

[8] E. Polak and D. Q. Mayne, "A Feasible Directions Algorithm for Optimal Control Problems with Control and Terminal Inequality Constraints", IEEE Trans. Automat. Control AC-22 (1977), 741-751.

[9] H. R. Sirisena, "Computation of Optimal Controls Using a Piecewise Polynomial Parametrization", IEEE Trans. Automat. Control AC-18, (1973), 409-411.

[10] H. R. Siresena and F. S. Chou, "Computation of Constrained Optimal Controls Using Parametrization Techniques", IEEE Trans. Automat. Control AC-19 (1974), 431-433.

[11] K. L. Teo and E. J. Moore, "On Directional Derivatives Methods for Solving Optımal Parameter Section Problems", Internat. J. Systems Sci. 9 (1978), 1029-1041.

[12] K. L. Teo and E. J. Moore, "Computational Methods for a Class of Time Delayed Optimal Control Problems", Nanta Mathematica 12 (1979), 203-221.

[13] K. L. Teo, K. H. Wong, and D. J. Clements, "Optimal Control Computation for Linear Time-Lag Systems with Linear Terminal Constraints", J. Optim. Theory Appl. 44 (1984), 509-526.

[14] K. L. Teo, K. H. Wong, and D. J. Clements, "A Feasible Directions Algorithm for Time-Lag Optimal Control Problems with Control and Terminal Inequality Constraints", J. Optim. Theory Appl. 46 (1985), 295-317.

[15] K. L. Teo and R. S. Womersley, "A Control Parametrization Algorithm for Optimal Control Problems Involving Linear Systems and Linear Terminal Inequality Constraints", Numer. Funct. Anal. Optum. 6 (1983), 297-313.

[16] K. L. Teo and Z. S. Wu, Computational Methods for Optimizing Distributed Parameter Systems (Academic Press, Orlando, 1984).

[17] K. H. Wong and K. L. Teo, "A Conditional Method for a Class of Time-Lag Optimal Control Problems", J. Austral. Math. Soc. Ser. B 25 (1984), 518-537.

[18] K. H. Wong, K. L. Teo and D. J. Clements, "Optimal Control Computation for Nonlinear Time-Lag Systems", J. Optim. Theory Appl. 47 (1985), 91-107. 